\title{
The Effect of Various ATM Switch Architectures on VBR Video Performance
}

\author{
Rose P. Tsang - rtsang@ca.sandia.gov Jenwei Hsieh and David H.C. Du \\ Sandia National Laboratories \\ Livermore, CA 94551 \\ University of Minnesota \\ Minneapolis, $M N 55455$
}

\begin{abstract}
One of the most important components of an Asynchronous Transfer Mode (ATM) network is the switch. Switch design is not a part of the ATM standards so vendors use a wide variety of techniques to build their switches. In this paper, we present experimental results of switching and multiplexing real-time Variable Bit Rate (VBR) digital video traffic (JPEG, MPEG-1, and MPEG-2) through two different ATM switch architectures. Real-time VBR traffic, such as digital video, is particularly interesting due to its high demands in terms of bandwidth, real-time delivery and processing requirements. Our experiments show that the 'fastest' switches, i.e., lowest latencies, do not necessarily perform better when transmitting VBR video. The impact of the high speed network components' characteristics, such as switch fabric architecture, buffering strategies, and higher layer transport protocols (i.e., UDP, TCP/IP), are illustrated through the experimental results.
\end{abstract}

\section{Keywords}

Asynchronous Transfer Mode (ATM), digital coded video, JPEG, MPEG, switch architectures, buffering strategies.

\section{INTRODUCTION}

One of the most important components of an Asynchronous Transfer Mode (ATM) network is the switch. An ATM switch must accept asynchronous and synchronous traffic, as well as connection-oriented and connection-less traffic. The speed at which a switch relays cells within a pre-specified cell loss probability bound is considered crucial; queueing and switching delay must be minimized. The switch processor must also be capable of handling high speed ports and aggregate throughputs of at least a couple gigabits per second.

Do high switch throughputs and low switch latencies translate into superior performance for all traffic types? The ATM standard is expected to serve as the transport mode for a wide spectrum of traffic types with varying performance requirements. In this study, we are interested in how well ATM switches can support real-time Variable Bit Rate (VBR) 
traffic. We present experimental performance results of real-time VBR traffic, namely digital compressed video, over two different ATM switch architectures. The experimental results are followed by a discussion of the results in relation to the capabilities and features of the switch architectures and network environment. We are interested in digital video because it is expected to be one of the predominant type of multimedia traffic transmitted on future high speed networks [Cole (1993), Pancha (1994)]. Video requires the continuous periodic delivery of data in order for the user not to perceive a degradation in the service quality (e.g., jerkiness due to the erratic delivery of video frames). Some data loss may be tolerated but the data must be delivered within a bounded amount of time. This is considered to be one of the most difficult data types to transport due to its real-time requirements as well as its dynamic variability of bandwidth requirements [Cole (1993), Tsang (1996)].

The type of video quality expected by today's users require very large network transmission speeds. Uncompressed speeds of broadcast-quality NTSC video and studio quality NTSC video are about $120 \mathrm{Mbits} / \mathrm{sec}$ and $216 \mathrm{Mbits} / \mathrm{sec}$, respectively [Cole (1993), Lyles (1992)]. Fortunately, recent advances in compression techniques [Cole (1993), LeGall (1991), Wallace (1991)] make the transmission of these high quality video media feasible. These compression techniques take advantage of the characteristics (or limitations) of the human visual system, to achieve lossy, yet visually lossless, compressed images and video. The two main video compression standards are commonly known as Joint Photographic Experts Group (JPEG) [Cole (1993), Wallace (1991)] and Moving Picture Experts Group (MPEG) [Cole (1993), LeGall (1991), Pancha (1994)]. JPEG is a still-image compression standard which can produce visually lossless compression with ratios up to 10 to 1 . The MPEG motion video standard was designed to support full motion video at compression ratios up to 200 to 1 . Bandwidth requirements for MPEG-2 range from 4 to 15 megabit per second, for a stream of $640 \times 480 \times 24$ pixels per frame ( 24 bit color), 30 frames per second digital NTSC-quality video [Cole (1993)].

The goals of our study were the following:

- We sought to measure the ability of an actual ATM local area network to support real-time variable bit sources, i.e., bursty periodic data. Real-time variable bit rate traffic tolerates a small degree of loss but strict timing requirements on frame delivery must be met. Moreover, frame sizes may vary greatly even between consecutive frames. Previous work on the performance of packet switch networks to support variable bit rate sources have used either analytical means or simulation models to predict performance [Heffes (1986), Norros (1991)]. However, accurate analytical models which capture the time-varying correlated nature of the stochastic process that model video streams are usually intractable [Maglaris (1988)]. It is also obvious that even in simulation models it is not possible to capture all aspects (or even most) of an actual distributed system. In a distributed system, there exist many components, hardware and software, whose complex interactions cannot be naturally captured or predicted by a fixed model.

- It is likely that video transmission will be one of the dominant media types involved in distributed multimedia systems. Most non-trivial video-based multimedia applications entail the network support of many multiplexed coded video streams. Given an ATM environment, with a known maximum achievable throughput and delay, how many 
typical coded video streams (e.g., MPEG-1, MPEG-2, JPEG) can be supported within reasonable loss and jitter bounds?

- We sought to determine the effect of switch architecture on the performance of digital video transmission. The Fore Systems ASX-200 and Cisco A100 Hyperswitch switch architectures provided an interesting basis for comparison of ATM switches architectures. Both switches rely upon Time Division Multiplexed (TDM) shared buses as the connecting switch fabric between input and output ports. However, both implement very different buffering strategies. We will present the effect of these different buffering strategies in our experiments.

- The Transmission Control Protocol (TCP/IP) and User Datagram Protocol (UDP/IP) suite of protocols are widely used today, and are very likely, during the initial deployment of ATM networks, to remain the dominant suite of protocols used in local and wide area network computing. How well is each suited for transmitting multiplexed video streams?

\section{TESTBED ENVIRONMENT}

The testbed environment consists of the following components:

Eight IBM RISC System/6000 (RS/6000) workstations. Each RS/6000 has a 66 $\mathrm{MHz}$ POWER2 processor. The operating system used was AIX 3.2.5. Each RS/6000 was equipped with an IBM Turboways 100 ATM Adapter (TURBOWAYS adapter) [IBM (1994)]. The TURBOWAYS adapter uses an onboard $25 \mathrm{MHz}$ Intel $\mathrm{i} 960$ processor. The adapter provides dedicated $100 \mathrm{Mbps}$ full-duplex connectivity using PVCs, and Direct Memory Access (DMA) capabilities.

A Fore Systems Forerunner ASX-200 ATM switch. The ASX-200 local ATM switch is Fore System's successor to the ASX-100 switch. Like the ASX-100, it is is based on a 2.4 Gigabits per second switch fabric (TDM bus) and a RISC control processor. The Fore ASX-200 switch architecture provides output buffering but no input buffering (see Figure 1).

Its features include output buffers with a 13,312 ATM cell capacity (per 2 output ports), dual leaky bucket policing, 'smart' buffers (per-VC queueing, multiple service priorities, dynamic buffer allocation), packet level discard and congestion control through the Explicit Forward Congestion Indicator.

A Cisco A100 Hyperswitch. The Hyperswitch is also based upon a 2.4 Gigabit per second TDM bus-based switch fabric. Its cells are routed through a combination of input and output buffers (see Figure 1). The input buffer size (per port) is 2450 ATM cells and the output buffer size (per port) is 50 ATM cells. Cells traverse through the switch fabric via a credit scheme which controls the admission of cells from the input buffers to the output buffers. In credit-based schemes, cells are never admitted to a network component unless that component is forwarded a 'credit' [Fan (1994)]. In the Hyperswitch, cell loss never occurs at the output buffers because before a cell may be admitted to the output buffer, the output buffer must send a signal (or credit) to the input buffer to let it know that it has available buffer slot(s). The Hyperswitch's switch architecture was based upon the "Expandable ATOM switch (XATOM)" design proposed at NEC [Fan (1994)]. 


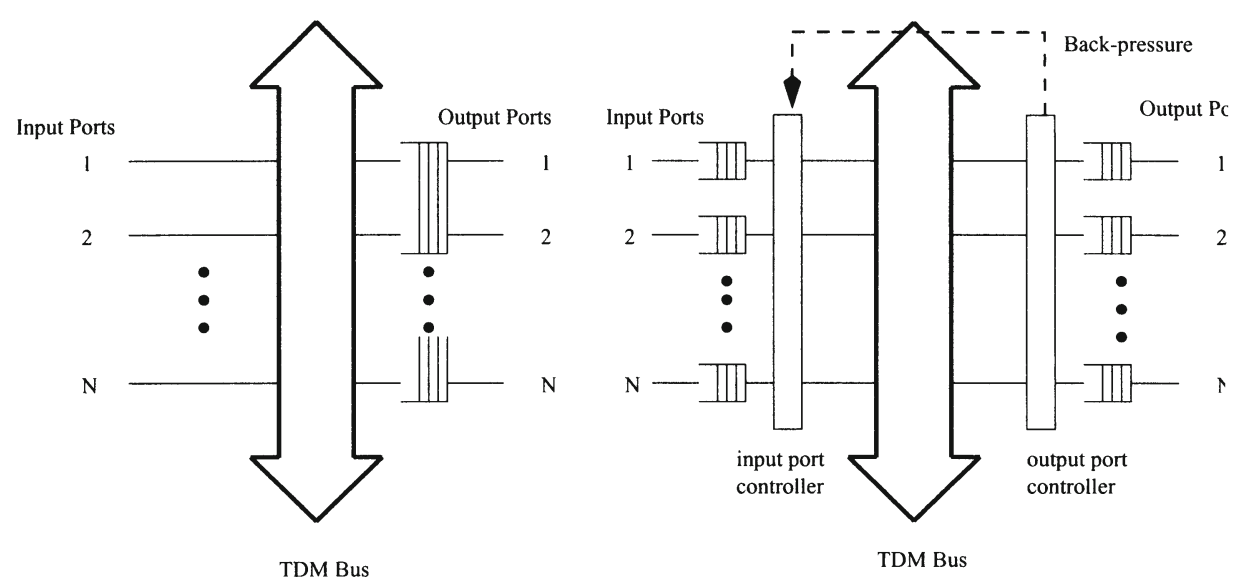

(a) Fore ASX-200 switch architecture

(b) Cisco A100 Hyperswitch switch architecture

Figure 1 Switch Architectures

100 Mbits/sec TAXI links interconnecting the RS/6000 hosts to the ATM switch. The topology of our experimental testbed consisted of an ATM switch (either the Fore or the Cisco) with direct TAXI connections to the $8 \mathrm{RS} / 6000$ hosts. Connectivity beween hosts was via one hop through the ATM switch.

The video data consisted of the following:

MPEG. The input video stream for the MPEG codec was a 3 minute 40 second digitized from laser disc with a frame resolution (similar to NTSC broadcast quality) of $512 \times 480$ pixels. This particular Star Wars sequence was chosen because it contained a mix of high and low action scenes. The interframe to intraframe ratio was 16 . The quantizer scale was 8. For these parameters, the image quality was judged to be good (constant) through the entire sequence of frames. The coded video was captured at 24 frames/second. The mean bit rate for the MPEG-1 sequence was approximately $1.5 \mathrm{Mbits} / \mathrm{sec}$; the bit rate varied from about $0.3 \mathrm{Mbits} / \mathrm{sec}$ to $4.5 \mathrm{Mbits} / \mathrm{sec}$. The mean bit rate for the MPEG-2 sequence was approximately $5.0 \mathrm{Mbits} / \mathrm{sec}$; the bit rate varied from about $0.8 \mathrm{Mbits} / \mathrm{sec}$ to 10.5 Mbits/sec.

JPEG. The input video stream for the JPEG codec was also a sequence from the Star Wars movie. The movie was input from a VCR and processed by a video card from Parallax Graphics, Inc., inside the Sparc 2. The frame resolution was $512 \times 480$ pixels. The quantization factor was 400 . The image quality was judged to range from fair to marginal. The coded video was captured at 15 frames/second. The mean bit rate was approximately $2.3 \mathrm{Mbits} / \mathrm{sec}$; the bit rate varied from about $2.0 \mathrm{Mbits} / \mathrm{sec}$ to $2.5 \mathrm{Mbits} / \mathrm{sec}$.

It is important to note that the input sequence we chose contains high as well as low motion scenes. Hence the performance results cannot be compared to all types of video 


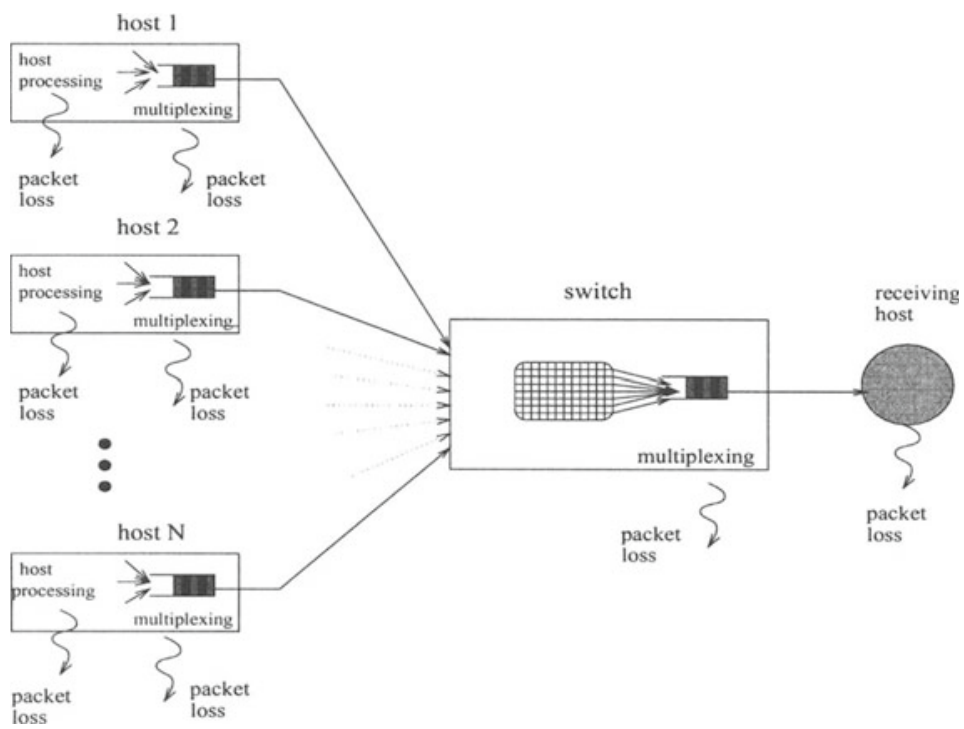

Figure $2 \mathrm{~N}$ senders to 1 receiver configuration

sequences. For instance, a video sequence generated from a video-conferencing application would contain, on average, smaller frames, since in most video-conferencing sequences there is little motion and few, if any, scene changes. Thus, better performance would be expected if such a sequence were used. We chose a video sequence with a high-level of motion in order to stress test the ATM network's ability to support bursty data.

We used the following configurations to measure how well the Fore ASX-200 switch and the Cisco Hyperswitch handled contention at the switch fabric, i.e., cross-traffic ( $\mathbf{N}$ senders to $\mathbf{N}$ receivers configuration), and contention at the output ports ( $\mathbf{N}$ senders to 1 receiver).

- $\mathbf{N}$ senders to 1 receiver. In this configuration, N RS/6000s simultaneously injected multiple video streams through the switch to a single receiver. See Figure 2.

- $\mathbf{N}$ senders to $\mathbf{N}$ receivers. In this configuration, $\mathrm{N} R \mathrm{RS} / 6000$ s simultaneously injected multiple video streams through the switch to another disjoint set of N RS/6000s. See Figure 3 .

The following terminology and performance metrics are used throughout the remainder of the paper.

The average send time of a frame is the time the first packet of a frame is transmitted to the time the first packet from the next consecutive frame is transmitted. When transmitting video frames at 24 frames per second, the average send time is $1 / 24=41.67$ milliseconds. The received interval is the time between the the receipt of an entire frame (the last packet in a frame) and the receipt of the next entire frame. This metric is used to compute jitter - the interarrival delay between consecutive frames. The fixed frame 


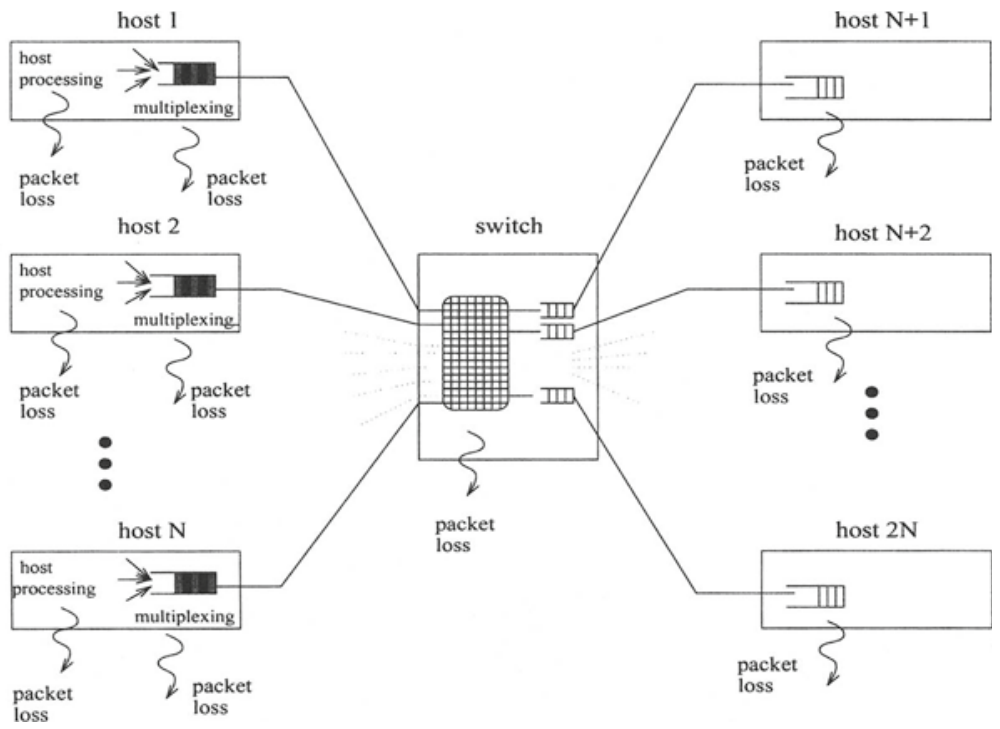

Figure $3 \mathrm{~N}$ senders to $\mathrm{N}$ receivers configuration

interval is the interval based upon the frame rate, e.g., 24 frames per second implies a fixed frame interval of $1 / 24=41.67$ milliseconds. In a jitter-free environment, all of the received intervals would be equal to the fixed frame interval. The percentage of lost frames is the percentage of lost frames during a single run, or averaged among several single runs. Any frame missing a packet is considered lost.

Data Collection. In both configurations (Figures 2 and 3), the sender(s) and receiver maintained logs of packet departures and arrivals. The sending host recorded the number of packets sent, and the average send time. If the sending host was transmitting $N$ streams (at 24 frames per second) and the average send time was larger than $1 / 24=42.67$ milliseconds, this situation implies that the sending host could not support the transmission of $N$ video streams at the 24 frame per second rate, i.e., the sending host was overloaded, and hence was introducing jitter which was not created by the network components. In these cases, the data was not used in this study.

On the receiving side, the receiving host receives the packets and demultiplexes them according to sender host id and port number. A log for each incoming video stream is kept. The number of the missing frames is recorded. Any frame missing a packet is considered lost. The time to receive each entire frame is recorded. The time between the the receipt of an entire frame and the receipt of the next entire frame is recorded.

For all our experiments, segments were 'played' for 3 minutes and 40 seconds. The data was 'played' by reading a file which consisted of an enumeration of frames and their corresponding byte sizes. To ensure accuracy, individual runs were executed multiple times. When appropiate, replicated results were averaged and the standard deviation computed and reported. 


\begin{tabular}{|c|c|c|}
\hline Test & Fore ASX-200 & Cisco Hyperswitch A 10 \\
\hline 7.488 Mbps CBR stream switched across one port & 10.57 microsecs & 39.90 microsecs \\
\hline $\begin{array}{l}7.488 \text { Mbps CBR stream plus bursty IP data to } \\
10 \% \text { capacity switched across the same port }\end{array}$ & 27.19 microsecs & 52.67 microsecs \\
\hline $\begin{array}{l}7.488 \text { Mbps CBR stream plus burst IP data to } \\
50 \% \text { capacity switched across the same port }\end{array}$ & 77.89 microsecs & 130.21 microsecs \\
\hline
\end{tabular}

Figure 4 Data collected at the ENL[16]: Latency per port under various loads.

\section{EXPERIMENTS}

\subsection{Previous Related Work}

A previous study [Mandeville (1995)] has shown the Fore ASX-200 to outperform the Cisco Hyperswitch in terms of latency and jitter (see Figure 4). This was not a surprising result considering the additional complexity of the Cisco Hyperswitch's buffering scheme. One purpose of this section is to see whether the additional functionality (credit-based inputoutput port buffering scheme), and hence overhead, as provided by the Cisco Hyperswitch, will serve to facilitate, or serve as an additional drawback, on the performance of VBR video transmission. Comparing the Fore Systems ASX-200 with the Cisco Hyperswitch performance is particularly illuminating since the ASX-200 provides only output buffering.

\subsection{Performance Measurements}

Experiment \#1: JPEG, MPEG-1, MPEG-2. In this experiment, the test configuration used was the 4 sender to 1 receiver model. The protocol suite used was UDP AAL5 ATM. The average (among all streams in the same run) percentage of frames lost as a function of the number of streams injected by the sending host is shown in Figure 5 . JPEG, MPEG-1, and MPEG-2 sequences from the Star Wars movies are compared. Despite the poorer quality JPEG video sequence and the lower capture rate of 15 frames/sec (compared to 24 frames/sec for the MPEG-1 sequence), the percentage of lost frames for the JPEG coded sequence was still higher than the percentage of lost frames for the MPEG-1 coded sequence (see Figure 5). The higher loss rates of the JPEG streams can be attributed to the following. The average frame size in the JPEG coded sequence consisted of 10944 Bytes. The average frame size in the MPEG-1 coded sequence consisted of 5184 Bytes. The average 'I' frame size in the MPEG-1 sequence was close to 11000 Bytes (which is close to the average frame size from the JPEG sequence). A sequence with an average larger frame size would result in greater losses because (i) any cell or packet loss would result in the entire frame being lost (i.e., smaller frames are less likely to be lost than larger frames), and (ii) a stream with a higher bit rate would cause more contention 


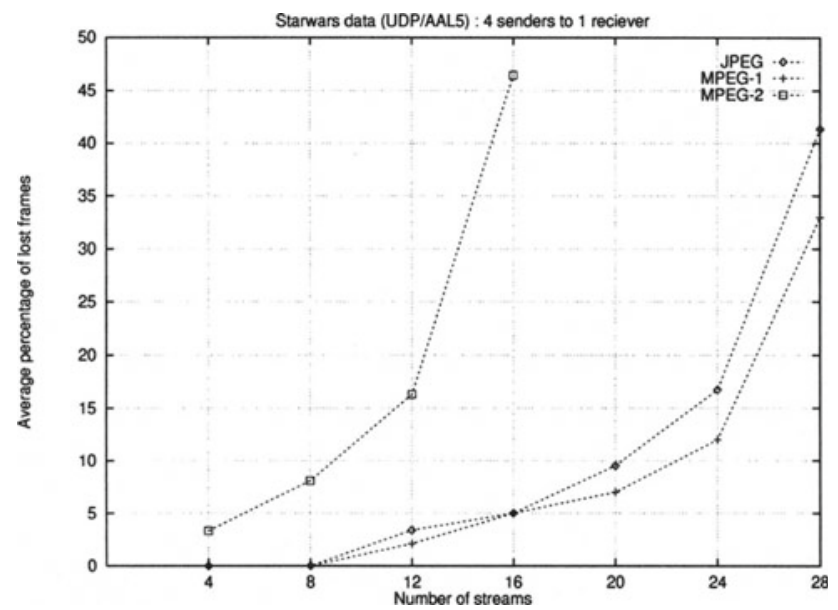

Figure 5 Experiment \#1: Frame loss rates for JPEG, MPEG-1, and MPEG-2

throughout its transmission and hence packets from that stream would be more likely to be discarded. For the same reason, due to the much larger frames in the MPEG-2 stream, the average percentage of lost frames for the MPEG-2 sequence was the greatest.

For the remainder of our experiments, we used the MPEG-1 sequence from the Star Wars movie. We chose to use the MPEG-1 sequence rather than the JPEG sequence because it is the most likely type of coded video to be used in the future; MPEG-1 provides higher compression ratios and lower mean bit rates than JPEG. The MPEG-2 stream data bit rate was too high (i.e., caused too many lost frames) to produce meaningful results in our particular environment. Thus, hereafter, the term MPEG implies MPEG-1.

As mentioned before, we used the TCP/IP and UDP/IP protocols as the transport mechanisms for transporting the video streams.

'Acceptable' visual quality. Continuous media traffic, such as coded video, have the real-time requirement that frames be displayed sequentially (continuously) with no prolonged delays between any pair of consecutive frames; the interarrival time, or jitter, between frames must be bounded.

When network congestion occurs, frames may be discarded (via buffer overflow), or in the case of TCP, may be discarded and then re-transmitted. If a frame arrives late, it will cause jerkiness in the visual medium. If a frame never arrives (i.e., is lost) its absence will also cause jerkiness in the visual medium. In MPEG, which consists of ' $\mathrm{I}$ ', 'P', and 'B' frames, some frames are more important than others [LeGall (1991), Tsang (1996)]. 'I' frames are complete bit images. They must be received regularly in order to re-generate high quality images. ' $\mathrm{P}$ ' and ' $\mathrm{B}$ ' frames are used to 'refresh' the current image. If a ' $\mathrm{P}$ ' or ' $\mathrm{B}$ ' frame is lost, the decoder may be able to 'guess', or estimate, the lost frame until the next ' $\mathrm{I}$ ' frame arrives. ' $\mathrm{I}$ ' frames serve as a reference point for creating ' $\mathrm{B}$ ' and 'P' 


\begin{tabular}{|c|c|c|c|c|c|c|c|c|}
\hline \multirow{2}{*}{$\begin{array}{l}\text { number of } \\
\text { streams }\end{array}$} & \multicolumn{4}{|c|}{ Fore ASX-200 } & \multicolumn{4}{|c|}{ Cisco Hyperswitch } \\
\hline & $\max$ & $\min$ & avg & std dev & $\max$ & $\min$ & avg & std dev \\
\hline \multicolumn{9}{|c|}{4 senders to 1 receiver } \\
\hline $\begin{array}{l}32 \text { streams } \\
(8 \text { / sender })\end{array}$ & $94.10 \%$ & $34.92 \%$ & $72.25 \%$ & 15.3 & $79.35 \%$ & $2.32 \%$ & $45.18 \%$ & 11.0 \\
\hline $\begin{array}{l}28 \text { streams } \\
\text { ( } 7 \text { / sender) }\end{array}$ & $75.02 \%$ & $1.71 \%$ & $32.71 \%$ & 12.5 & $58.10 \%$ & $2.49 \%$ & $22.50 \%$ & 9.1 \\
\hline $\begin{array}{l}24 \text { streams } \\
(6 / \text { streams })\end{array}$ & $33.83 \%$ & $1.60 \%$ & $11.81 \%$ & 10.2 & $21.42 \%$ & $1.46 \%$ & $5.00 \%$ & 2.6 \\
\hline $\begin{array}{l}20 \text { streams } \\
\text { ( } 5 \text { / sender) }\end{array}$ & $24.80 \%$ & $0.93 \%$ & $6.74 \%$ & 3.6 & $5.45 \%$ & $0.32 \%$ & $1.95 \%$ & 0.8 \\
\hline $\begin{array}{l}16 \text { streams } \\
\text { ( } 4 \text { / sender) }\end{array}$ & $9.35 \%$ & $0.74 \%$ & $5.32 \%$ & 2.3 & $1.49 \%$ & $0.26 \%$ & $0.98 \%$ & 0.5 \\
\hline \multicolumn{9}{|c|}{2 senders to 1 receiver } \\
\hline $\begin{array}{l}28 \text { streams } \\
(14 / \text { sender })\end{array}$ & $56.9 \%$ & $1.42 \%$ & $21.32 \%$ & 11.6 & $42.9 \%$ & $1.48 \%$ & $18.21 \%$ & 7.3 \\
\hline $\begin{array}{l}24 \text { streams } \\
(12 / \text { sender })\end{array}$ & $21.0 \%$ & $1.40 \%$ & $9.36 \%$ & 5.6 & $14.1 \%$ & $1.68 \%$ & $4.63 \%$ & 1.5 \\
\hline $\begin{array}{l}20 \text { streams } \\
(10 / \text { sender })\end{array}$ & $16.01 \%$ & $0.73 \%$ & $6.87 \%$ & 4.8 & $3.6 \%$ & $0.33 \%$ & $1.99 \%$ & 1.3 \\
\hline $\begin{array}{l}16 \text { streams } \\
(8 / \text { sender })\end{array}$ & $5.43 \%$ & $0.25 \%$ & $3.79 \%$ & 1.6 & $1.12 \%$ & $0.0 \%$ & $1.02 \%$ & 0.4 \\
\hline
\end{tabular}

Figure 6 Experiment \#2: $\mathrm{N}$ senders to 1 receiver: frame loss comparisons for dif ferent switches

frames. Hence, 'I' frames are more important to maintaining high visual quality; their loss is much more apparent to a viewer.

In our experiments, we define a loss of more than $10 \%$ to be visually noticeable to most viewers and hence unacceptable. The worst case occurs when all $10 \%$ of the lost frames are 'I' frames. At 24 frames per second, a $10 \%$ loss translates into a 21.6 frame per second frame rate. This is in the range of what is usually judged to be 'acceptable' visual quality.

Experiment \#2: Output Port Contention Measurements In this experiment, the test configuration used was the $\mathrm{N}$ sender to 1 receiver model (see Figure 2). The protocol suite was UDP AAL5 ATM.

Figure 6 depicts the two test scenarios. In the first scenario, we transmitted multiple video streams from 4 sending hosts to 1 receiving host. In the second scenario, we transmitted multiple video streams from 2 sending hosts to 1 receiving host.

This test was performed in order to measure how well each switch handled contention 


\begin{tabular}{|c|c|c|c|c|c|c|c|c|}
\hline \multirow{2}{*}{$\begin{array}{l}\text { number of } \\
\text { streams }\end{array}$} & \multicolumn{4}{|c|}{ Fore ASX-200 } & \multicolumn{4}{|c|}{ Cisco Hyperswitch } \\
\hline & $\max$ & $\min$ & avg & std dev & $\max$ & $\min$ & avg & std dev \\
\hline $\begin{array}{l}128 \text { streams } \\
\text { (32 sender) }\end{array}$ & $92.92 \%$ & $48.1 \%$ & $73.46 \%$ & 12.1 & $76.91 \%$ & $2.19 \%$ & $40.25 \%$ & 14.2 \\
\hline $\begin{array}{l}112 \text { streams } \\
\text { (28 sender) }\end{array}$ & $70.81 \%$ & $36.3 \%$ & $53.32 \%$ & 9.9 & $68.89 \%$ & $3.14 \%$ & $23.82 \%$ & 11.3 \\
\hline $\begin{array}{l}96 \text { streams } \\
(24 \text { sender })\end{array}$ & $22.26 \%$ & $1.8 \%$ & $10.08 \%$ & 5.0 & $23.32 \%$ & $0.7 \%$ & $9.69 \%$ & 4.1 \\
\hline $\begin{array}{l}80 \text { streams } \\
\text { ( } 20 \text { sender) }\end{array}$ & $13.16 \%$ & $1.0 \%$ & $2.55 \%$ & 1.8 & $3.18 \%$ & $0.4 \%$ & $1.15 \%$ & 0.4 \\
\hline
\end{tabular}

Figure 7 Experiment \#3: $\mathrm{N}$ senders to $\mathrm{N}$ receivers: frame loss comparisons for different switches

at an output port. Examining Figure 6, the first item to note is that given the same number of streams, for both the Fore and Cisco switch, the 2 senders to 1 receiver case has a consistently lower frame loss rate than the 4 senders to 1 reveiver case. Given the same number of streams, while only varying the number of senders, implies that for a greater number of senders there is more contention at the output port and hence greater frame loss; for a fewer number of senders, more streams are already superimposed (multiplexed) at each sending host and less at the output port of the switch.

The comparison between how well the Cisco A100 Hyperswitch and Fore Systems ASX200 switch handled output contention shows that the Cisco switch is able to consistently lose fewer frames than the Fore ASX-200 switch. Again, this may be attributed to there being less contention at the output port of the Cisco switch than the Fore switch. Recall, that the Cisco switch uses an input-output credit-based buffering scheme where loss never occurs at the output ports; data is only sent to an output port if that output port has sent a credit to the input port, where the data is being held, signaling that it has a certain amount of empty buffer space. Thus in the Cisco switch, contention is distributed among the input ports whereas in the Fore switch (for the $\mathrm{N}$ sender to 1 receiver case) all the contention occurs at a single output port.

Experiment \#3: Cross Traffic Measurements. In this experiment, the test configuration used was the $\mathrm{N}$ sender to $\mathrm{N}$ receiver model (see Figure 3 ). The protocol suite was UDP AAL5 ATM.

In this experiment, we transmitted multiple video streams between disjoint sets of 4 sending hosts and 4 receiving hosts, i.e., 4 pairs of sending-receiving hosts. Each pair of hosts supported the transmission of multiple MPEG streams. Figure 7 depicts the frame loss percentages as a function of load for both the Cisco and Fore switches. It can be seen that the switch fabric of the Cisco switch is able to handle the same traffic (as was handled by the Fore switch) but with a consistently lower percentage of lost frames. Again this may be attributed to the more sophisticated buffering scheme provided by the Cisco Hyperswitch. In the Cisco switch, contention is distributed among the input ports first 


\begin{tabular}{|c|c|c|c|c|c|c|}
\hline \multirow{2}{*}{$\begin{array}{l}\text { number of } \\
\text { streams }\end{array}$} & \multicolumn{3}{|c|}{ Fore ASX-200 } & \multicolumn{3}{|c|}{ Cisco Hyperswitch } \\
\hline & {$[2,4)$} & {$[4,6)$} & {$[6$, infinity $)$} & {$[2,4)$} & {$[4,6)$} & {$[6$, infinity $)$} \\
\hline $\begin{array}{l}32 \text { streams } \\
(8 / \text { sender })\end{array}$ & $21 \%$ & $13 \%$ & $7 \%$ & $17 \%$ & $12 \%$ & $5 \%$ \\
\hline $\begin{array}{l}28 \text { streams } \\
\text { ( } 7 \text { / sender) }\end{array}$ & $17 \%$ & $6 \%$ & $3 \%$ & $14 \%$ & $7 \%$ & $2 \%$ \\
\hline $\begin{array}{l}24 \text { streams } \\
\text { (6/ sender) }\end{array}$ & $15 \%$ & $4 \%$ & $3 \%$ & $12 \%$ & $5 \%$ & $0 \%$ \\
\hline $\begin{array}{l}20 \text { streams } \\
(5 / \text { sender })\end{array}$ & $10 \%$ & $3 \%$ & $0 \%$ & $6 \%$ & $1 \%$ & $0 \%$ \\
\hline
\end{tabular}

Figure 8 Experiment \#4: 4 senders to 1 receiver - percentage delayed

whereas in the Fore switch all the contention occurs at the switch fabric (TDM bus) immediately.

Experiment \#4: Jitter vs. number of multiplexed streams. This experiment was performed using TCP on ATM AAL5.

Figure 8 depicts the percentages of frames delayed for varying numbers of fixed frame intervals. Recall, that loss is not a performance measure in this experiment since TCP rarely loses packets. Packets which are delayed between $x$ fixed frame intervals and $y-1$ fixed frame intervals are said to be delayed by $[x, y)$, i.e., their received interval is between $[x, y)$.

Using TCP, packets which are discarded by the network are re-transmitted. Thus packets (and hence frames) are very rarely lost. Packets are discarded only after a pre-set number of re-transmissions occur. However, this situation occurs very rarely, and we did not observe any loss for any of the TCP experiments. We assumed that the receiving host could buffer up to 3 fixed frame intervals (or 3 frames). This is a reasonable assumption because ' $\mathrm{B}$ ' frames may reference either forward or backward frames; both the last and next ' $\mathrm{P}$ ' or ' $\mathrm{I}$ ' frames must be transmitted before the ' $\mathrm{B}$ ' frames may be sent. Thus the receiver host must be able to buffer a minimal of 3 frames. Hence a frame received within 3 fixed frame intervals will not cause jitter to occur. A frame which arrives outside of 3 fixed frame intervals is considered lost (undisplayable) and may contribute to the overall perceived jitter. Thus, similarly to what is acceptable for lost frames, we define an untolerable number of delays (i.e., losses) to be $10 \%$; if more than $10 \%$ of the frames are delayed (beyond 3 fixed frame intervals), the visual quality is unacceptable.

Assuming frame delays less than or equal to 3 fixed frame intervals are tolerable (due to buffering), TCP can support up to (28, Fore switch) and (30, Cisco switch) multiplexed streams with only $9 \%$ of the received intervals exceeding 3 fixed frame intervals.

Note that TCP is able to support a far greater number of multiplexed streams (within reasonable inter-arrival delay constraints) than UDP (within reasonable frame loss rates). Also for TCP the variation in jitter between individual multiplexed streams from the 
same run was found to be negligible. Recall that for UDP the variation in frame loss rates between individual multiplexed streams from the same run was very high.

\section{DISCUSSION OF RESULTS}

Recently many new distributed multimedia applications have been proposed. These new applications are based upon the transmission of digital information such as coded video, voice, high-resolution images, data, and graphics. For the network provider, the challenge lies in providing adequate network support for these various data types in order to operate on a communications infrastructure with a shared switching and transmission facility.

From our results, we conclude with the following points of discussion.

Fast packet switching? Much emphasis has been placed on the need for fast packet switches as a means of enabling the high transport speeds of ATM [Biagioni (1993), Fan (1994)]. This need for fast packet switches has brought about the philosophy that switches with the simplest, yet efficient, architectures are the most desireable. The ideal architecture which has been cited is one which provides either no input buffering (or an input buffering scheme which does not have the 'head-of-line' blocking problem) and buffers at each output port [Biagioni (1993), Mandeville (1995)]. This appears to have been the philosophy behind the switch architecture of the Fore Systems ATM switches. And, indeed, the Fore Systems switch does provide lower jitter and delay than most other available ATM switches including the Cisco Hyperswitch. This was shown in [Mandeville (1995)].

How do a few microsecond jitter differences, or even a few hundred microsecond jitter differences translate into the application Quality of Service (QoS) for video transmission, i.e., frame inter-arrival time of 30-40 milliseconds ? From the results in our experiments, we can infer that there is not a direct translation and (within reasonable bounds) the jitter (latency) differences and end-to-end performance of video need not even be related.

From our experimental results, as well as the results in [Mandeville (1995)]. we reason that the Cisco switch performed superiorly because it uses a more sophisticated buffering scheme (at input and output ports) which uses a form of flow control to 'smooth' the traffic between the input and output ports [Fan (1994)]; fewer cells are lost due to buffer overflow. The Fore switch uses pure output buffering so all contention must be resolved at the output buffer - hence a greater number of buffer overflows may occur. So in the end, it did not matter that the Cisco switch incurred more latency (jitter) than the Fore switch!

Network Transport Protocols. Due to the real-time nature of video traffic, a guaranteed service protocol, or a best-effort service protocol with real-time scheduling at individual switch nodes, has usually been suggested as the most appropiate type of protocol for multimedia traffic such as video. Protocols which provide end-to-end flow control and re-transmissions, such as TCP, have been considered unsuitable because it has usually been thought that the delays caused would result in packets missing their bounded delay requirements and hence becoming meaningless (discarded). From our experiments, we observed TCP's sliding window protocol to have the effect of smoothing traffic burstiness, as well as still being able to deliver packets within their deadlines. Thus our results 
showed that the appropiate type of flow control may greatly improve application level performance significantly over a best-effort transport mechanism. In our particular environment TCP worked very well. This is not to suggest TCP as an appropiate video transport layer. Actually, in other environments (e.g., a wide-area network), it is very unlikely TCP would perform as well. The important point is that explicit flow control, either or a combination of link-link or end-end mechanisms, must be used to improve the performance of real-time traffic such as video. Best-effort delivery, in conjunction with real-time scheduling at individual switch nodes, which has been widely suggested as the most appropiate transport mechanism for real-time traffic such as video, is unlikely to be adequate.

Traffic Control. Traffic control for VBR video traffic is difficult to implement due to the real-time nature of the traffic. But, we believe it to be a necessary measure to increase network utilization and provide more stringent guarantees to continuous media traffic.

When the hosts and the network are stressed, the experimental data show that the burstiness of the variable bit rate coded video streams is a significant factor in the resulting performance degradation. We saw that controlling burstiness (through end-end flow control or switch buffering techniques) results in significantly less packet losses. This implies a fewer number of re-transmissions for transport protocols which guarantee reliability through re-transmissions, such as TCP, as well as for unreliable transport protocols such as UDP. Losses are significant when transmitting traffic types with large pre-specified (non-changeable) frame sizes like coded video. If any packet of a frame is lost, the entire frame may be discarded. Thus a relatively small percentage of lost ATM cells may translate into a relatively large frame loss percentage in data traffic (such as coded video) which must use large frame sizes. Our results show that traffic control results in a significantly decreased frame loss rate while maintaining acceptable jitter and loss bounds.

\section{REFERENCES}

Biagioni, E., Coope, E., Samsom, R. (1993) Designing a Practical ATM LAN. IEEE Network, March 1993.

Cole, B. (1993) The Technology Framework. IEEE Spectrum, March 1993.

Fan, R., Suzuki, H., Yamada, K., Matsuura, N. (1994) Expandable ATM Switch Architecture (XATOM) for ATM LANs. CISCO Systems Journal.

Heffes, H., Lucantoni, D. (1986) A Markov Modulated Characterization of Packetized Voice and Data Traffic and Related Statistical Multiplexer Performance. IEEE Journal on Selected Areas of Communication, September 1986.

IBM (1994) TURBOWAYS 100 ATM Adapter: User's Guide. March 1994.

Le Gall, D. (1991) MPEG: A Video Compression Standard for Multimedia Applications. Communications of the ACM, April 1991.

Lyles, J., Swinehart, D. (1992) The Emerging Gigabit Environment and the Role of Local ATM. IEEE Communications Magazine, April 1992.

Maglaris, B., Anastassiou, D., Sen, P., Karlsson, G., Robbins, J. (1988) Performance Models of Statistical Multiplexing in Packet Video Communications. IEEE Transactions on Communications, July 1988.

Mandeville, R. (1995) The ATM Stress Test: Which Switches Survived ? Data Commu- 
nication, March 1995.

Norros, I., Roberts, J., Simonian, A., Virtamo, J. (1991) The Superposition of Variable Bit Rate Sources in an ATM Multiplexer. IEEE Journal on Selected Areas in Communications, April 1991.

Pancha, P., El Zarki, M. (1994) MPEG Coding for Variable Bit Rate Video Transmission. IEEE Communications Magazine, May 1994.

Tsang, R., Pavan, A., Du, D. (1996) Experiments with Video Transmission over an Asynchronous Transfer Network. ACM/Springer-Verlag Multimedia Systems Journal, August 1996.

Wallace, G. (1991) The JPEG Still Picture Compression Standard. Communications of the ACM, April 1991. 